\title{
PERSPECTIVAS DA ELIMINAÇÃO DA HANSENÍASE NO ESTADO DE SÃO PAULO E NO BRASIL
}

\author{
PROSPECTIVES FOR ELIMINATION OF LEPROSY IN THE STATE OF SÃO PAULO AND IN BRAZIL
}

Wagner Nogueira ${ }^{1} \&$ Mary Lise Carvalho Marzliak ${ }^{2}$

\begin{abstract}
${ }^{1}$ Coordenador do Programa de Controle de Hanseníase; ${ }^{2}$ Médica - Divisão Técnica de Hanseníase/Controle e Vigilância Epidemiológica - Secretaria de Estado da Saúde de São Paulo.

CORRESPONDÊNCIA: Wagner Nogueira - Rua São Luís, 99 / 6º andar - 01046-001 - São Paulo-SP - Fone (Fax) 011-231-0689.
\end{abstract}

NOGUEIRA W \& MARZLIAK MLC. Perspectivas da eliminação da hanseníase no Estado de São Paulo e no Brasil. Medicina, Ribeirão Preto, 30: 364-370, jul./set. 1997

RESUMO: Partindo da meta estabelecida pela Organização Mundial de Saúde e referendada pelo Brasil, em 1991, da eliminação da lepra como problema de saúde pública mundial pelo ano 2000, são analisados, neste artigo, os progressos alcançados pelo programa de controle da hanseníase no país e, particularmente, no Estado de São Paulo. Apesar da nítida redução da prevalência da doença pela adoção de poliquimioterapia e dos progressos no seu diagnóstico, os altos níveis de abandono de tratamento e, possivelmente, de ocorrência de recidivas, podem comprometer o objetivo de eliminação nacional da hanseníase a curto prazo.

UNITERMOS: Hanseníase. Saúde Pública.

\section{1 - INTRODUÇÃO}

A hanseníase é um importante problema de saúde pública e, durante muitos séculos, a inexistência de terapêutica eficaz contribuiu para isto.

0 advento da Sulfona, no início da década de 40 , trouxe grande alento à comunidade científica, uma vez que se acreditou possível o controle desta doença. Foram realizadas grandes mudanças nas políticas de controle da doença, e o tratamento através do isolamento compulsório foi abolido, sendo substituído pelo tratamento em regime ambulatorial.

Contudo, a inexistência de uma rede de serviços, funcionando adequadamente, passou a se constituir em grande entrave na luta contra esta endemia e, apesar da descoberta de novas drogas, como a Clofa- zimina e a Rifampicina, nas décadas de 60 e 70, respectivamente, muitos pesquisadores começaram a evidenciar a resistência medicamentosa à monoterapia sulfônica como resultado do uso irregular deste esquema terapêutico.

Os esquemas terapêuticos, recomendados no final da década de 70, passaram a incluir a Rifampicina para os doentes multibacilares e a monoterapia sulfônica para os doentes paucibacilares, em períodos de tratamento de longa duração (no mínimo 5 anos para os multibacilares e 2 anos para os paucibacilares). Assim, até então, milhares de casos eram diagnosticados no mundo, anualmente, e iam sendo somados aos casos dos anos anteriores, resultando num acúmulo de casos registrados, o que implicou no aumento do coeficiente de prevalência, ano a ano. 
No início da década de 80, a Organização Mundial de Saúde passou a recomendar o uso do esquema poliquimioterápico, associando a Rifampicina ao tratamento monoterápico dos doentes paucibacilares, e a Clofazimina, ao tratamento dos doentes multibacilares, que, também, passaram a receber a Rifampicina em doses intermitentes, durante todo o tratamento. $\mathrm{O}$ tempo de tratamento preconizado passou a ser de seis meses para os paucibacilares e de dois anos para os multibacilares, sendo portanto, bastante reduzido.

A redução do tempo de tratamento e o conceito de cura, muito embora encontrando algumas resistências na comunidade científica, passaram a ser adotados pela maioria dos países, com o aval do Comitê de Terapêutica da Organização Mundial de Saúde, e, assim, uma nova realidade na história secular desta endemia começa a ser escrita, observando-se a inversão do fluxo de entradas e saídas do registro ativo de doentes, evidenciando-se, anualmente, um número maior de saídas por cura do que de entradas, com conseqüente redução do número de doentes registrados, bem como queda do coeficiente de prevalência.

No final da década de 80 , a maioria dos países com endemia já havia implantado o esquema poliquimioterápico. O Brasil passou a fazê-lo a partir de 1990, após ter desenvolvido grandes esforços na reorganização de serviços, em anos anteriores como parte da estratégia nacional para a implantação deste esquema terapêutico, capacitando pessoal em massa, investindo em equipamentos e insumos necessários para a implementação, em todo o país, da rede de serviços de ações de controle da hanseníase.

Em 1991, durante a $44^{\text {a }}$ Conferência Mundial de Saúde, após a avaliação dos resultados obtidos com a implantação da poliquimioterapia, os países membros comprometeram-se a eliminar a hanseníase como problema de saúde pública até o ano 2000, definindo-se eliminação como a redução do coeficiente de prevalência para menos de 1 doente, a cada 10.000 habitantes. Nesta Conferência, o Brasil estava representado, passando, assim, a ser signatário deste compromisso.

\section{2 - PRINCIPAIS RESULTADOS DO PROGRAMA DE CONTROLE DA HANSENÍASE}

O número de casos registrados no mundo, segundo informações da Organização Mundial de Saúde, era de 2.850.000, em 1966, passando para 3.600.000, em 1976, e para 5.400.000 casos em 1985. A partir de então, este número começou a cair, chegando a 3.200.000 casos em 1991 e 926.259 casos em 1996. Notamos, assim, a inversão da tendência ao acúmulo de casos a partir da metade da década de 80 , resultado do uso, em larga escala, dos esquemas poliquimioterápicos, da redefinição do tempo de tratamento, bem como em decorrência dos critérios adotados para alta por cura.

No Brasil, o número de casos registrados foi aumentando até o ano de 1990, passando a declinar a partir de 1991, conforme pode ser observado na Figura 1.

Assim, a inversão, observada no mundo a partir de 1987, somente aconteceu no Brasil a partir de 1991, quando a poliquimioterapia foi implantada em massa, cabendo reiterar que este atraso se deveu, principalmente, aos grandes investimentos, a nível nacional, na reorganização dos serviços e na estratégia de implantação gradual, a partir de projetos-piloto distribuídos por todo o país.

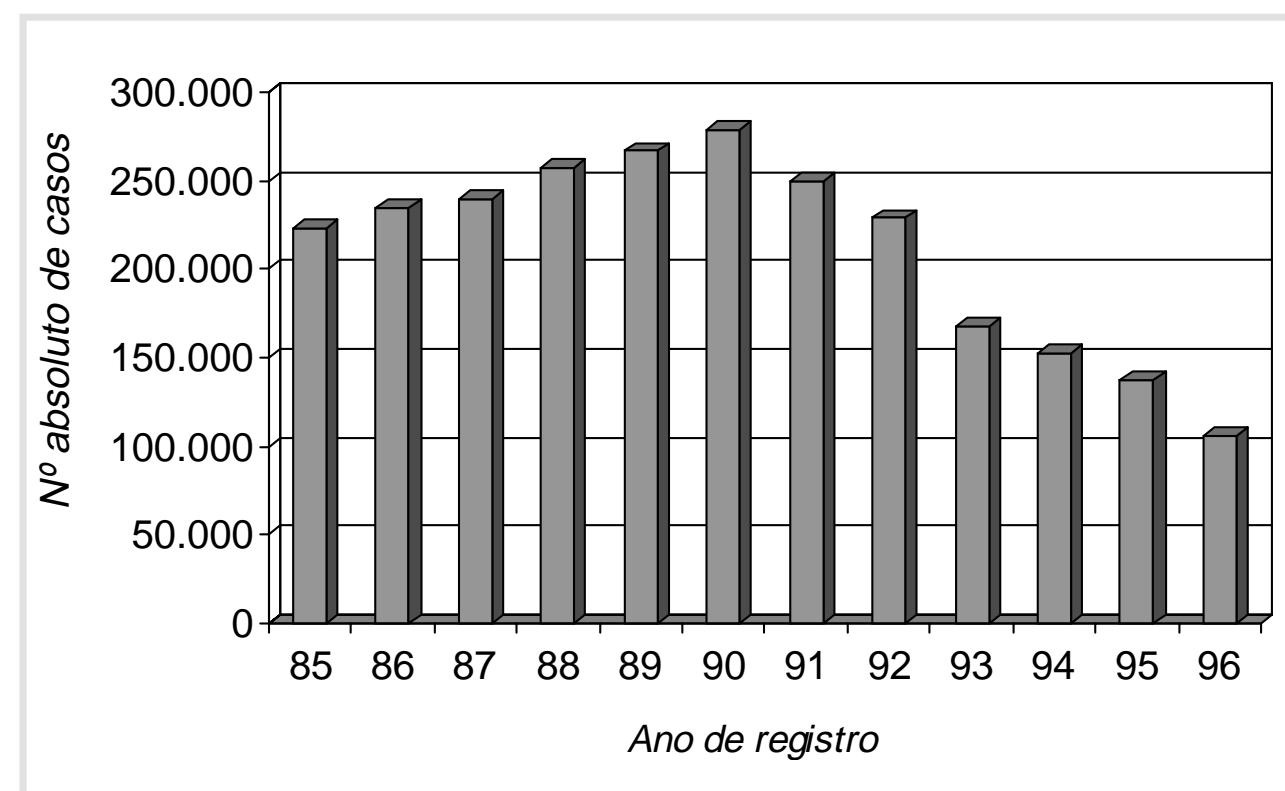

Figura 1 - Número de casos de hanseníase, registrados no Brasil entre 1985 e 1996. 
A redução do número absoluto de casos registrados refletiu-se nos coeficientes de prevalência, que também começaram a diminuir a partir de 1991, como pode ser observado na Figura 2.

Cabe destacar que, neste período, houve importante incremento na detecção de casos, como pode ser visto na Figura 3, evidenciando, assim, uma melhora na capacidade operacional de diagnóstico, muito provavelmente decorrente dos esforços desenvolvidos na reorganização dos serviços em todo o território nacional.

Portanto, a redução dos coeficientes de prevalência encontra justificativa não na diminuição do número de casos diagnosticados, mas, sim, no aumento do número de altas por cura, como podemos observar na Figura 4. Observamos a redução gradativa da proporção de saídas do registro ativo por outros motivos (óbitos, transferências, altas estatísticas) e o aumento da proporção de altas por cura, a partir do ano de 1987, com maior intensidade a partir de 1991. No ano de 1996, a proporção das saídas por outros motivos tem um aumento devido às mudanças de critério de alta estatística, reduzindo de 10 para 5 anos para os doentes multibacilares e de 5 para 2 anos para os doentes paucibacilares. Assim, no ano de 1996, com a aplicação deste novo critério, subiu a proporção de saídas por outros motivos, sem que isto significasse mudança de tendência, anteriormente observada.

No Estado de São Paulo, o número absoluto de doentes registrados no período de 1975 a 1990 apresentou discreta tendência ao crescimento, muito embora tenha apresentado flutuações neste mesmo período. A partir de 1991, observamos uma diminuição acentuada, conforme é mostrado na Figura 5.

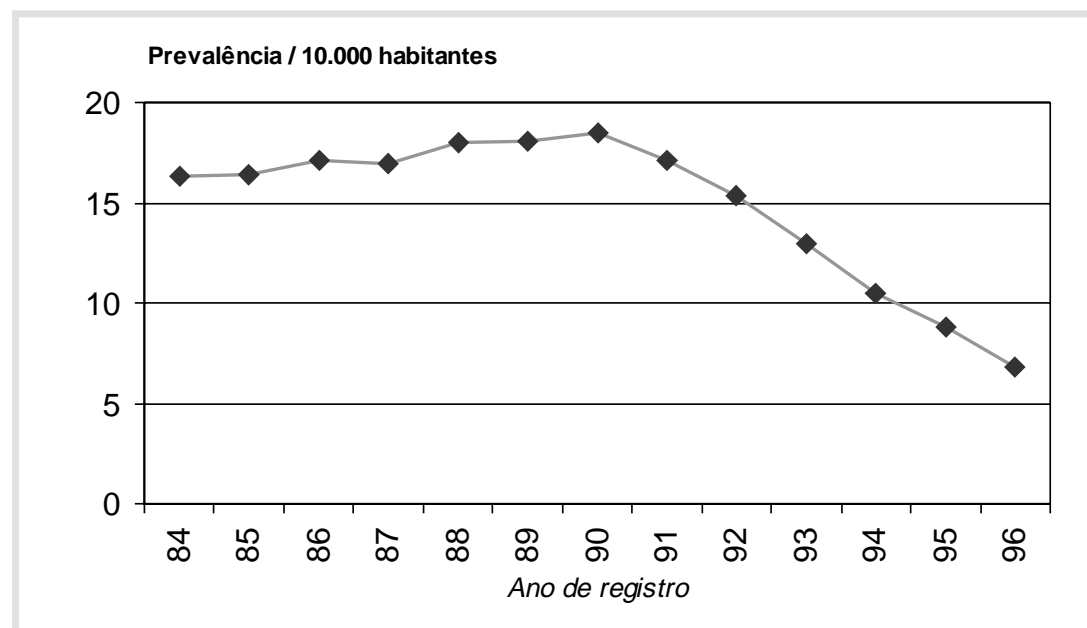

Figura 2 - Coeficientes de prevalência de hanseníase no Brasil, entre 1984 e 1996. FONTE: CNDS/CENEPI/FNS/MS

Obs.: Os Estados de Roraima e Mato Grosso do Sul não informaram dados.

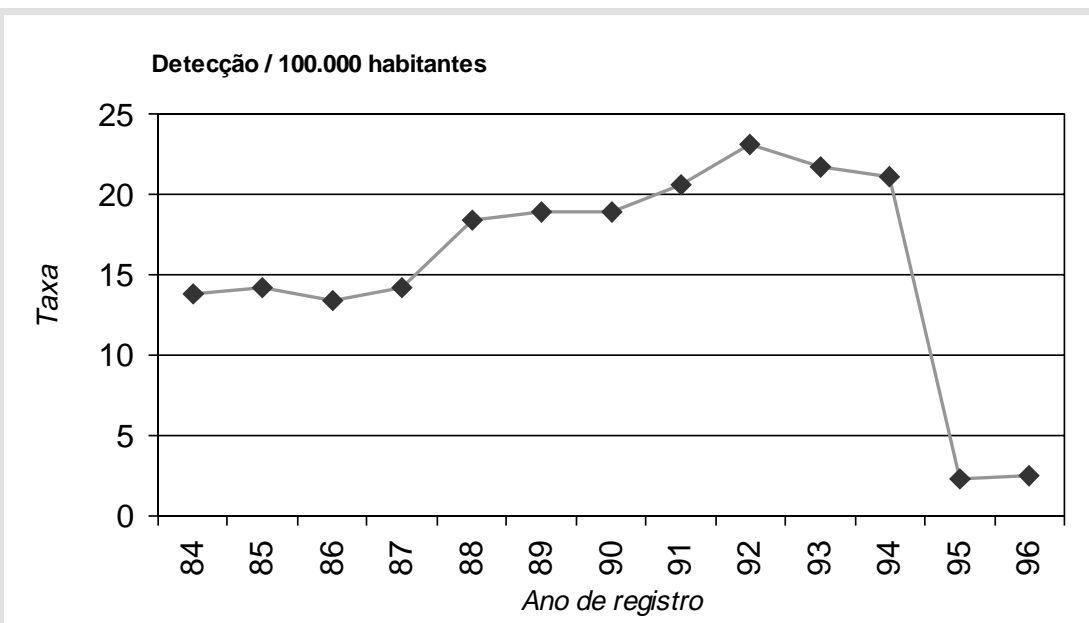

Figura 3 - Taxa de deteç̧ão de casos novos de hanseníase no Brasil, entre 1984 e 1996.

FONTE: Coordenação Nacional de Dermatologia Sanitária/Ministério da Saúde.

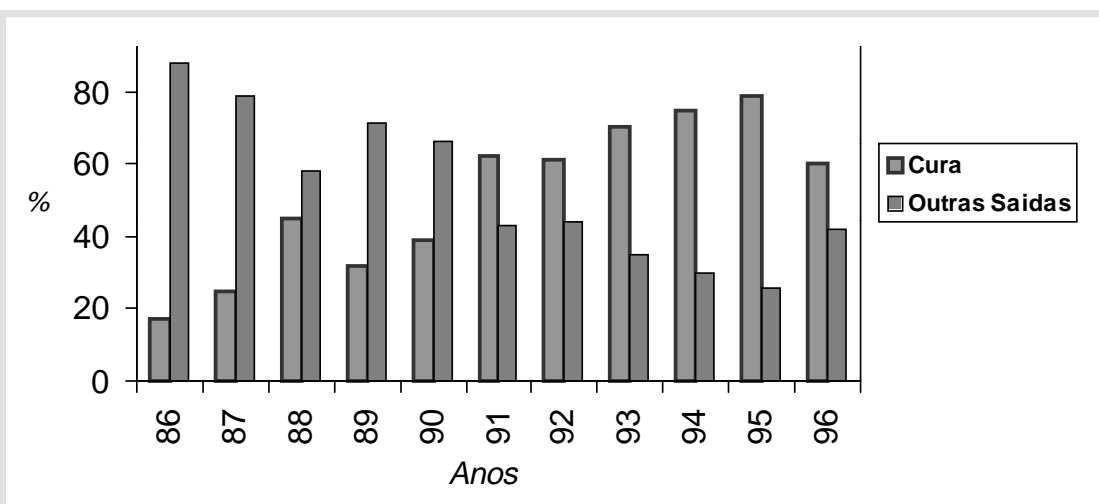

Figura 4 - Percentual de curas entre as saídas do Registro Ativo de Hanseníase no Brasil, entre 1986 e 1996.

FONTE: MS/FNS/CENEPI/CNDS 


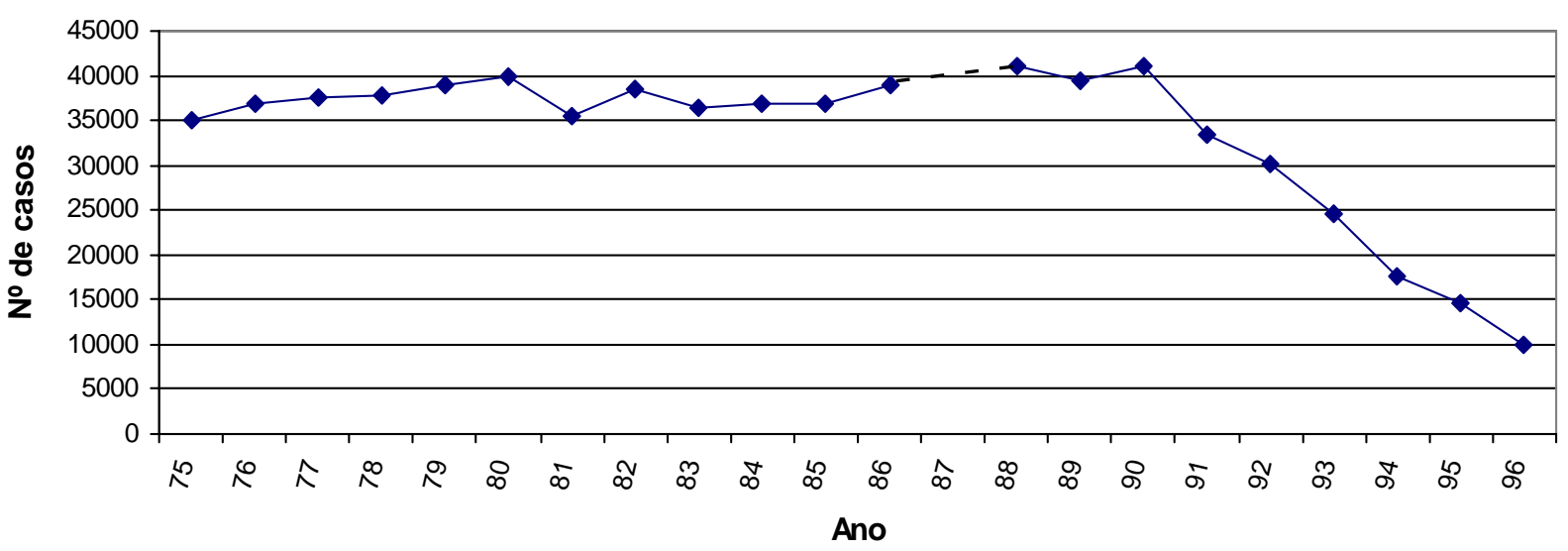

Figura 5 - Número absoluto de casos de hanseníase registrados no Estado de São Paulo de 1975 a 1996.

As repercussões de redução do número absoluto nos coeficientes de prevalência podem ser observadas na Figura 6, que inclui, além dos anos acima citados, todo o período de controle no Estado de São Paulo, ou seja, desde 1924, cabendo destacar que a série histórica, de 1924 a 1970, está contida na Tese de Doutoramento do Dr. Walter Belda, e o período de 1971 a 1996, a partir dos registros da Divisão Técnica de Hanseníase do Centro de Vigilância Epidemiológica do Estado de São Paulo.

Nesta série histórica, podemos observar que, a partir do final da década de 40 , tivemos uma redução no ritmo de crescimento da endemia, notando-se a inversão da tendência de crescimento a partir do final da década de 70, passando, a partir de então, a cair, gradativamente, até o ano de 1990, quando se acentua a diminuição dos coeficientes de prevalência dos anos que seguem, mostrando que, neste Estado, a endemia já vinha apresentando diminuição dos coeficientes de prevalência, até mesmo antes da implantação do esquema poliquimioterápico.

Observando-se a detecção nesta mesma série histórica, na Figura 7, notamos que apresentou tendência nitidamente crescente no período de 1924 a 1942 ,

\section{Coeficiente de Prevalência da Hanseníase no Estado de São Paulo, periodo de 1924 a 1996.}

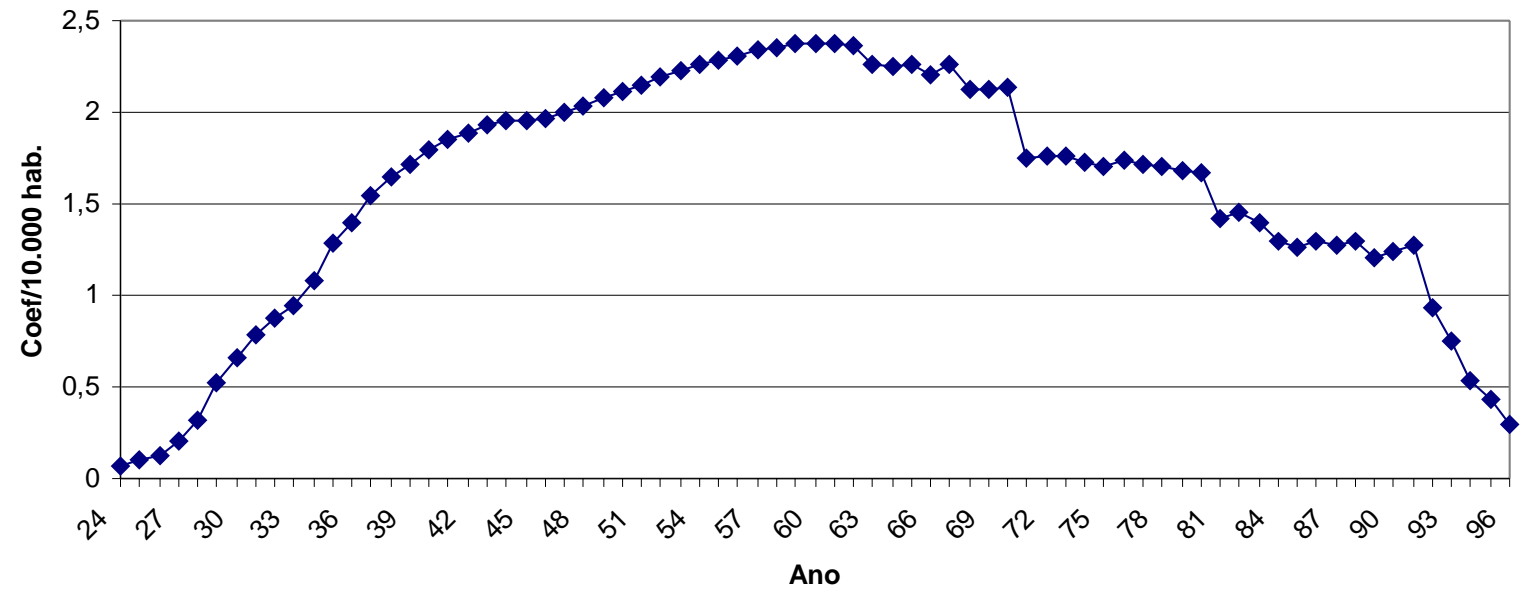

Figura 6 - Prevalência da hanseníase no Estado de São Paulo, no período de 1924 a 1996. 


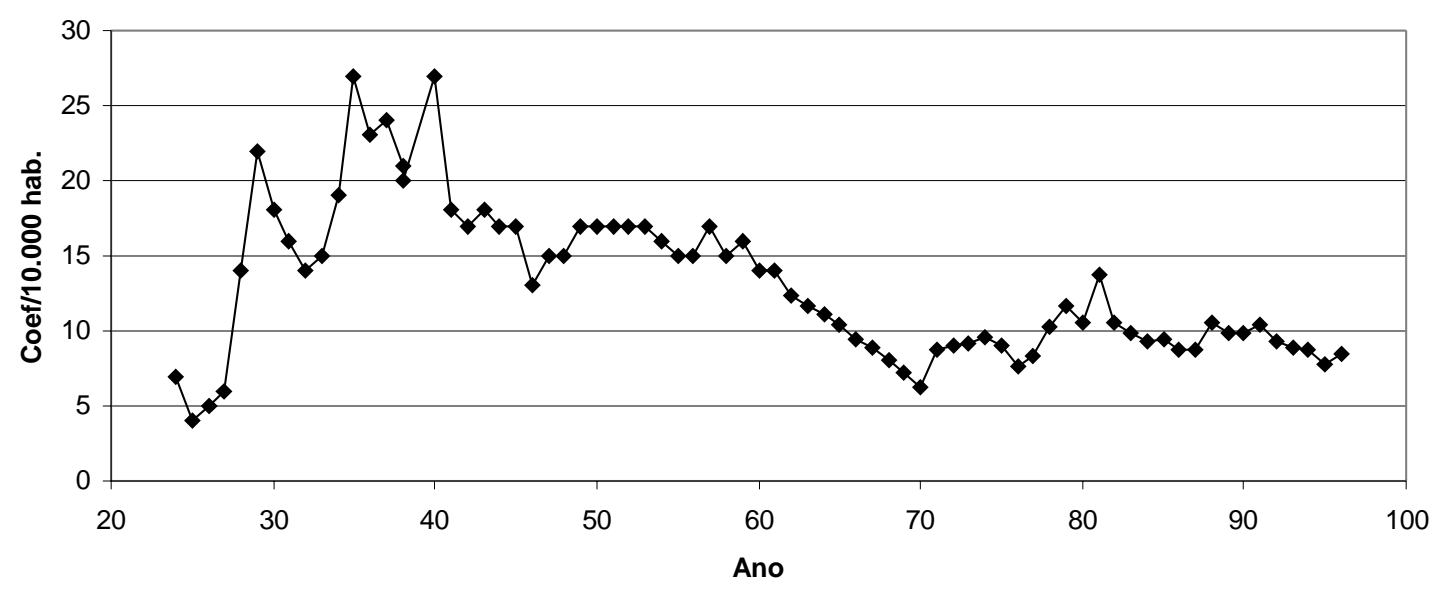

Figura 7 - Taxas de detecção de hanseniase no Estado de São Paulo, período de 1924 a 1996.

passando a tendência decrescente de 1943 a 1970 (Tese do Dr. Walter Belda), e, a partir de 1971, muito embora não se disponha da curva de tendência, a série histórica evidencia um discreto aumento da detecção, permitindo-nos inferir uma provável melhora na capacidade operacional de detecção e creditar a queda de prevalência ao incremento do número de altas por cura, o que pode ser confirmado na Figura 8, que mostra o número total de saídas e, dentre estas, o total de altas por cura.

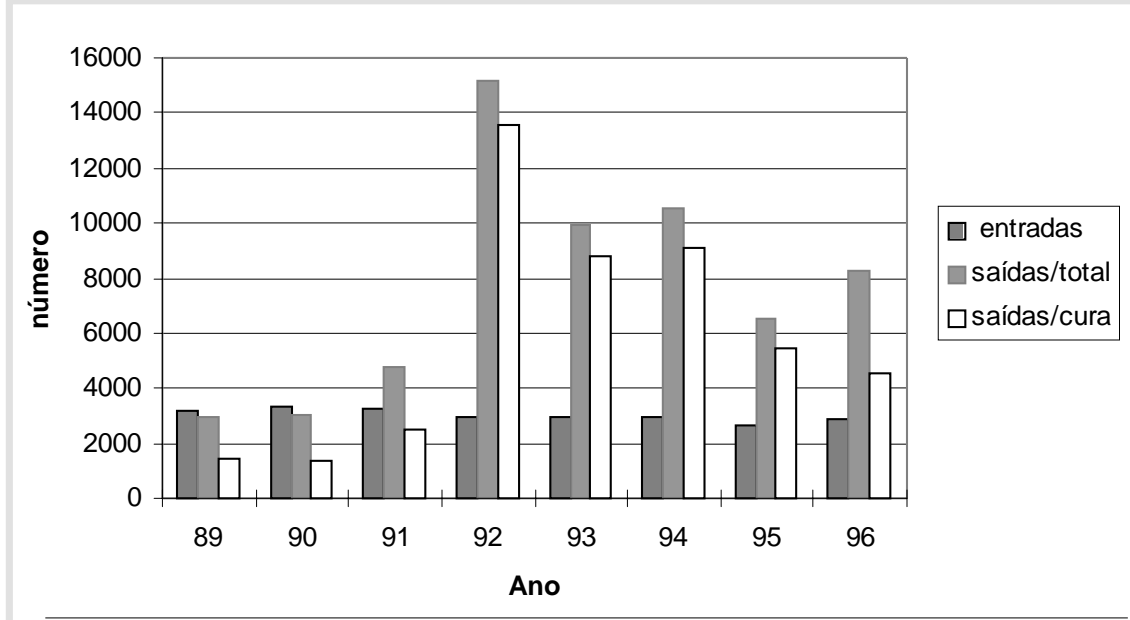

Figura 8 - Número total de entradas, total de saídas e saídas por cura, do registro ativo, do Estado de São Paulo - 1989 a 1996.
Observamos que, a partir de 1991, houve importante incremento no número de doentes retirados do registro ativo, mais acentuado nos anos subseqüentes; de 1992 a 1996, e que o principal motivo foi a alta por cura, totalizando, nestes cinco anos, 41.550 pacientes, contra uma entrada de 14.359 pacientes no mesmo período.

O elevado número de altas por cura, que vem sendo observado não só no mundo como no Brasil e também no Estado de São Paulo, vem de encontro às expectativas mundiais de viabilidade da eliminação da hanseníase como problema de saúde pública, e o Ministério da Saúde, a partir dos resultados observados a partir de 1986, elaborou uma projeção da prevalência até o ano 2000 (Figura 9).

Podemos observar uma esperança de redução da prevalência nacional para níveis próximos de 5 casos em cada 10.000 habitantes, longe ainda do nível recomendado para a eliminação, menos de 1 caso a cada 10.000 habitantes, todavia bem inferior ao coeficiente de 18,5 casos/10.000 hab. observado na década de 90.

Da mesma forma, ao analisarmos os últimos resultados auferidos, temos que a projeção da prevalência do Estado de São Paulo, para o ano 2000, gira ao redor de 6.000 casos, correspondendo a um coeficiente menor que 2 doentes a cada 10.000 habitantes, coeficiente este também bastante inferior à prevalência do início da década (11.6/10.000 hab.) mas, ainda, bem superior ao nível de eliminação recomendado pela Organização Mundial de Saúde. 


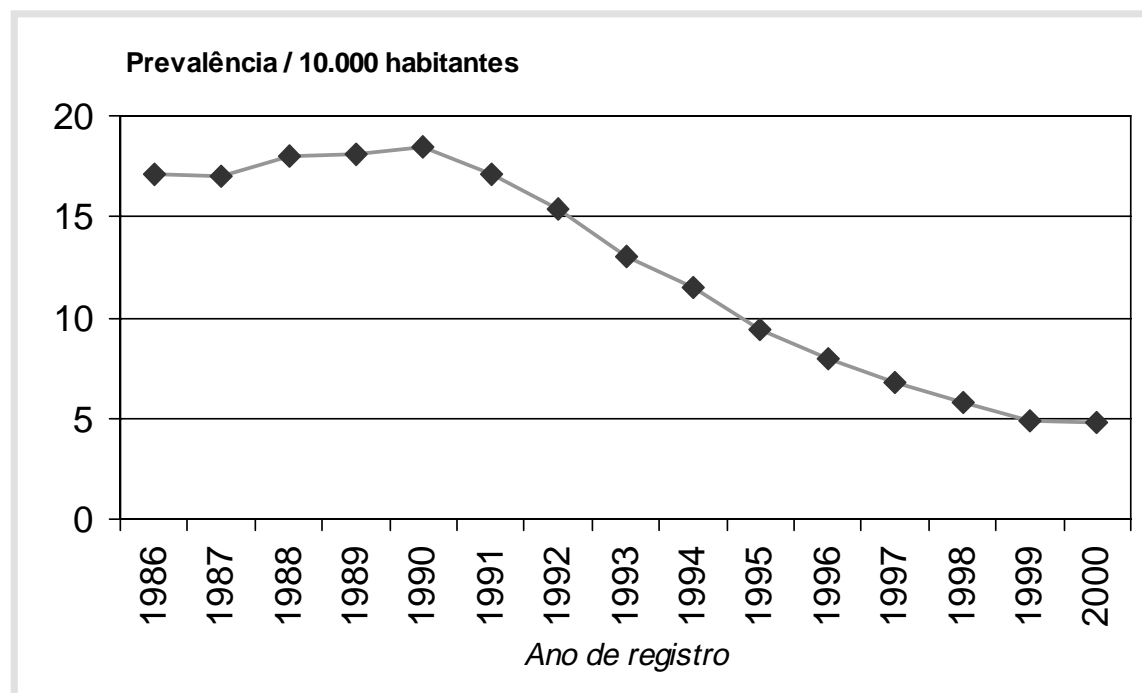

Figura 9 - Taxas de Prevalência Anual de Hanseníase no Brasil 1986 - 2000.

0 número de casos de doentes não atendidos/não informados no Brasil, no ano de 1996, foi de 30.121, correspondendo a $43 \%$ do total do registro ativo. Inclui todos os casos de abandono acumulados até 5 anos para os multibacilares e até 2 anos para os paucibacilares. Com a rápida redução do registro ativo, pela cura dos casos atendidos, a expectativa para o ano 2000 é de um elevado número de casos em abandono dentro do registro ativo, justificando o desenvolvimento de esforços no sentido da recuperação e tratamento desses casos.

\section{3 - PRINCIPAIS MOTIVOS QUE IMPEDEM A ELI- MINAÇÃO DA HANSENÍASE NO BRASIL}

Avaliando-se os resultados observados acima, a questão que se levanta está relacionada aos motivos pelos quais, a nível federal e estadual, considera-se a viabilidade de se alcançar a meta de eliminação da hanseníase enquanto problema de saúde pública.

A justificativa para este fato está principalmente relacionada aos critérios adotados pelo país para alta estatística, mantendo em registro, por 5 anos, os doentes multibacilares e, por 2 anos, os doentes paucibacilares. Desta forma, os casos em abandono vão se acumulando, ano após ano, contribuindo, assim, para coeficientes de prevalência mais elevados. Muitos países do mundo vêm adotando critérios diferentes, sendo que alguns retiram do registro os doentes em abandono, no final de cada ano, o que facilita bastante o alcance da meta de eliminação.

Estes procedimentos, normatizados para alta estatística, vêm sendo continuamente discutidos pelo Comitê Assessor da Coordenação Nacional de Dermatologia Sanitária e os critérios atualmente adotados vêm sendo referendados pela maioria dos integrantes deste Comitê, o que justifica sua adoção, principalmente considerando o período de incubação da doença e a necessidade do desenvolvimento de esforços operacionais, prevenindo a irregularidade do tratamento, a prevenção e a recuperação dos casos em abandono, até que a meta de eliminação seja alcançada efetivamente e não simplesmente às custas de subterfúgios estatísticos.
No Estado de São Paulo, em 1996, estavam em abandono 2.398 casos, representando $24 \%$ do registro ativo. Somente a detecção anual acrescida dos casos em abandono indica uma prevalência maior do que 1 caso em cada 10.000 habitantes, coeficiente superior ao nível de eliminação, para o Brasil e também para o Estado de São Paulo.

Assim, no Brasil e também em São Paulo, a viabilidade do alcance da meta de eliminação encontra como principal barreira a existência de um elevado número de casos em abandono. Esta meta somente será alcançada com a recuperação dos casos fora de controle, bem como com o aprimoramento das condições operacionais da rede de serviços, reduzindo a capacidade de produção de doentes em abandono que vem sendo verificada nos últimos anos.

Mantendo-se as condições operacionais atuais da rede de serviços do país e do Estado de São Paulo, a eliminação somente será alcançada com a mudança dos atuais critérios de alta estatística, apesar de que não parece ser esta a tendência da imensa maioria dos técnicos que coordenam as ações de controle no país e nos estados.

Finalizando, a utilização em massa dos esquemas de duração fixa de 6 a 24 doses em até 9 meses e 36 meses para os doentes pauci e multibacilares, respectivamente, vem contribuindo para a identificação de um novo problema para o alcance da meta de eliminação, relacionado à ocorrência de recidivas (vide capítulo V). Muitos técnicos de nível local vêm 
levantando suas preocupações, mesmo antes de qualquer comprovação científica que quantifique a ocorrência de recidivas na intensidade que muitos vêm propagando. Alguns técnicos elaboraram protocolos de investigação para o dimensionamento real deste fenômeno e, até que se obtenham resultados conclusivos, continuamos no campo da especulação.

Contudo, dependendo da intensidade com que ocorrem as recidivas, elas podem vir a se constituir em mais um entrave para o alcance da eliminação, uma vez que parte dos doentes considerados curados regressariam para o tratamento.

Assim, a meta de eliminação da hanseníase no Brasil encontra, no momento, como principais obstáculos, a irregularidade e o abandono do tratamento e a possibilidade das recidivas virem a compor o elenco de preocupações que podem inviabilizar o alcance desta meta.

NOGUEIRA W \& MARZLIAK MLC. Prospectives for elimination of leprosy in the State of São Paulo and in Brazil. Medicina, Ribeirão Preto, 30: 364-370, july/sept. 1997.

ABSTRACT: In this work, we analyze the advances reached by the Program of Leprosy Control in Brazil and in the State of the São Paulo, in view of the goal set up by WHO and supported by Brazilian authorities of eliminating leprosy as a public health problem by the year 2000 . In spite of the clear reduction of leprosy prevalence in Brazil by the adoption of polychemotherapy and of advances in the diagnosis of the disease, high levels of defection from treatment and, possibly, of relapses, may hint the goal of national elimination of leprosy in the near future.

UNITERMS: Leprosy. Public Health.

\section{BIBLIOGRAFIA CONSULTADA}

1 - BELDA W. A endemia da hanseníase no Estado de São Paulo. Tese de Doutorado, Faculdade de Saúde Pública da USP, 1974.

2 - BRASIL. Ministério da Saúde. Centro Nacional de Epidemiologia, Coordenação Nacional de Dermatologia Sanitária. Informações Epidemiológicas e Operacionais do Programa de Controle e Eliminação da Hanseníase, Brasília, 1996.

3 - BRASIL. Ministério da Saúde. Centro Nacional de Epidemiologia, Coordenação Nacional de Dermatologia Sanitária, Relatório de Avaliação Nacional do Controle da Hanseníase, 1994, 1995, 1996. Brasília, 1994/1996.

4 - BRASIL. Ministério da Saúde. Centro Nacional de Epidemiologia. Coordenação Nacional de Dermatologia Sanitária. Plano de Eliminação da Hanseníase no Brasil para o período de 1995 - 2000. Brasília, 1995.
5 - SECRETARIA DE ESTADO DA SAÚDE DE SÃO PAULO. Centro de Apoio ao Desenvolvimento das Ações Integradas de Saúde. Plano de Eliminação da Hanseníase para o período de 1995 - 1998. São Paulo, 1994.

6 - SECRETARIA DE ESTADO DA SAÚDE DE SÃO PAULO. Centro de Vigilância Epidemiológica "Prof. Alexandre Vranjac" Relatório da Endemia Hansênica no Estado de São Paulo, 1994, 1995, 1996. São Paulo, 1994/1996.

Recebido para publicação em 02/07/97

Aprovado para publicação em 30/07/97 